\title{
THE BUFFERED PERFORMIC-ACID-ALCIAN-BLUE- PERIODIC-ACID-SCHIFF METHOD FOR THE DIFFERENTIATION OF BASOPHILS IN THE HUMAN AND RAT PITUITARY
}

\author{
BY \\ K. SWETTENHAM \\ From the Bernhard Baron Institute of Pathology, the London Hospital
}

(RECEIVED FOR PUBLICATION FEBRUARY 24, 1960)

\begin{abstract}
The difficulty of using a single method for staining rat and human pituitary was investigated by testing separately and in different combinations the effects of the various components in the performic-acid-alcian-blue(P.F.A.B.)-periodic-acid-Schiff(P.A.S.) method (Adams and Swettenham, 1958). In this way the possibility of inhibiting or enhancing by one procedure the staining effects of the other could be assessed. The profound influence of fixation on these staining methods was demonstrated by employing the same set of staining methods on tissues fixed in different ways.

The possibility that unbuffered performic acid might exert a considerable solvent action on certain proteins was considered to be related to the difficulty of applying the P.F.A.B.-P.A.S. method to the rat pituitary. Buffered performic acid has far less solvent action and the P.F.A.B.P.A.S. method, modified by the replacement of buffered for unbuffered performic acid, proved to be successful in the demonstration of two types of basophil cells in both rat and man.
\end{abstract}

The P.A.S. method introduced by McManus (1946) and Hotchkiss (1948) was applied to the human pituitary by Everson Pearse (1949) and to the rat by Purves and Griesbach (1951). It appears to offer the considerable advantage that its effect depends on a predictable chemical reaction. With this method a mucoprotein component was identified in basophils but not in acidophils and thus a chemical differentiation between these cells was established. A further advantage of this type of method, based on a histochemical reaction, is that its application is not dependent on the use of regressive dyes, the differentiation of which depends on the skill of the operator. Some variability in staining reaction is, however, dependent on the effects of different fixatives (Everson Pearse, 1953a). Purves and Griesbach (1951) reported two types of P.A.S.-positive cell in the rat differing in regional distribution, relationship with blood vessels, and the shape and size of the cell and granules. These cells were stated to react selectively to moderate variations in the level of circulatory sex and thyroid hormones, and were called gonadotrophs and thyrotrophs respectively.
The gonadotrophs were described as coarsely granulated oval cells located mainly at the periphery of the gland close to blood vessels and often containing a conspicuous negative image of the Golgi apparatus. They considered that these cells responded to castration and developed a signet-ring appearance, the coalescent vacuoles displacing the nucleus to one side (Severinghaus, 1938).

A modification of the P.A.S. technique was introduced by Wilson and Ezrin (1954), the aim of which was to differentiate basophils into two types ("P.A.S.-red and P.A.S.-purple") in the anterior pituitary. This method is greatly dependent on variable counterstaining controlled by the operator ; it has enjoyed only temporary popularity and has been superseded by a further modification by Ezrin, Swanson, Humphrey, Dawson, and Wilson (1958).

Following the work of Romeis (1940), who described two types of basophil cells using resorcin-fuchsin with a modified Azan counterslain, Halmi (1950, 1951, 1952) described a method for the differentiation of two types of basophil cells in the rat's pituitary using the 
aldehyde-fuchsin method of Gomori (1950) with a modified azan counterstain. Those with an affinity for aldehyde-fuchsin (called beta cells) corresponded with the thyrotrophs of Purves and Griesbach, whilst those demonstrated only by azan (called delta cells) corresponded to the gonadotrophs.

The aldehyde-fuchsin method seems applicable only to the rat's pituitary since neither Fand (1955) nor Everson Pearse (1956) was able to obtain convincing results in human material. As Halmi and Davies (1953) described what appeared to be a partial reversal of results if acidified permanganate was used instead of iodine in the preliminary oxidation with the Halmi technique, the type of oxidation required may be of some importance. Thus the use of performic acid as the oxidizing agent with the aldehyde-fuchsin technique allows the demonstration of two types of basophil cells in the human pituitary (Russell, 1956).

The performic - acid - alcian - blue (P.F.A.B.) method for cystine (Adams and Sloper, 1956) coupled with the P.A.S. technique as a counterstain was applied to the human pituitary by Adams and Swettenham (1958). This has been stated to be unsuccessful in the rat (Adams and Everson Pearse, 1959), but, as will be shown, this is principally due to the failure of P.A.S. to act as an effective counterstain in these circumstances.

Danielli (1947) showed that performic acid had a considerable solvent effect on protein in most of the methods in which it was incorporated, and suggested that buffered performic acid was a greatly improved oxidizing agent in that its solvent effect was less. If Danielli's view can be applied to the rat pituitary, then this diminished solvent action may influence the results of staining by the P.F.A.B.-P.A.S. method. It therefore seemed reasonable to investigate the effects of using buffered performic acid as the oxidizing agent in this method.

The purpose of this work is to attempt to apply the buffered P.F.A.B.-P.A.S. method to both human and rat pituitaries, and to investigate the effects of fixation on the various dyes which are incorporated in this method.

\section{Materials and Methods}

The pituitaries from 24 normal male and female rats, weighing 170-300 g., were placed in different fixatives and serial sections $5 \mu$ thick were prepared. The sections were mounted in groups of 25 on five slides and subjected to five staining procedures. The sections were arranged in such a way that adjacent sections were stained by different procedures for the purposes of close comparison.
The pituitaries obtained from 24 patients post mortem were also fixed in different ways, cut serially and stained in the same way as the rat sections.

Fixatives.-The following were the fixatives used and the times for fixation:

(1) Cold $4 \%$ formaldehyde saline, five hours.

(2) $4 \%$ formaldehyde saline, 24 hours.

(3) $4 \%$ formaldehyde-sublimate-saline, 24 hours.

(4) $70 \%$ alcohol, 24 hours.

Stains.-The following were used :

(1) P.A.S. - orange G.

(2) Buffered performic acid-alcian blue (B.P.F.A.B.).

(3) P.F.A.B.

(4) Aldehyde fuchsin and Halmi.

(5) P.A.S. only.

(6) B.P.F.A.B.-P.A.S.

(7) P.F.A.B.-P.A.S.

Stain 2 was alternated in some cases with stain 6 and stain 3 with stain 7 .

\section{Rat Pituitary}

Results.-In the assessment of the techniques carried out in this work, attention is paid to these P.A.S.-positive cells, and their differentiation into Halmi's beta and delta types will be observed.

Fixation Effects.-It was found in the experiments in this series that positive P.A.S., P.F.A.B., and buffered P.F.A.B. staining was obtained only after fixation with formalin-containing reagents. Of these, the results for the P.A.S. reaction were best after fixation in cold formaldehyde saline, although the results after fixation in formaldehyde saline at room temperature were satisfactory in that the counterstain did not mask the reaction. The final preparations in this latter group were not as vivid as when the temperature of fixation was low. After formaldehyde-sublimate-saline fixation the positive P.A.S. staining was not quite as strong as with formol saline.

The best results for the buffered and unbuffered P.F.A.B. method were obtained after the use of formaldehyde saline and formaldehyde-sublimatesaline fixation for 24 hours. The results in both buffered and unbuffered P.F.A.B. methods were unreliable after fixation in cold formaldehyde saline.

No staining was obtained with the P.A.S. buffered or unbuffered P.F.A.B. or aldehydefuchsin methods after fixation with $70 \%$ alcohol.

In this series, counterstaining with orange $G$ and haematoxylin did not obscure the P.A.S. reaction. This and the aldehyde-fuchsin and P.F.A.B. methods all gave consistent results with formaldehyde saline and formaldehyde-sublimatesaline fixation. 


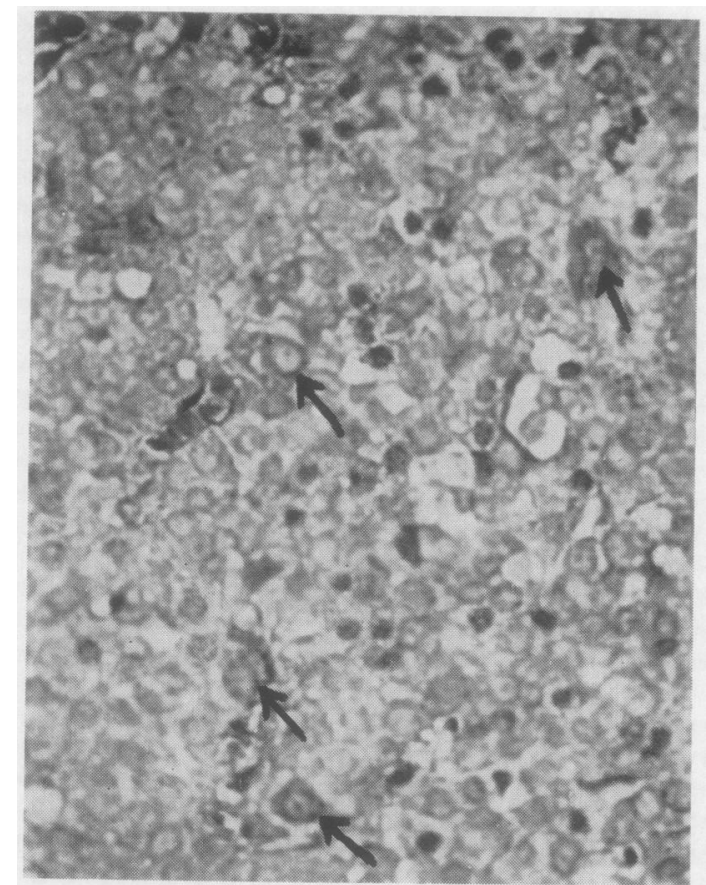

FIG. 1.-Rat pituitary to show lack of staining of basophils (arrows) 1 after pretreatment with unbuffered performic acid. P.A.S. $\times 540$.

Results with P.A.S. Reaction Used as Counterstain to P.F.A.B. Method.-Sections stained by the P.F.A.B. method were counterstained by the P.A.S. reaction (Adams and Swettenham). It was possible to study the effect of the P.F.A.B. method on the demonstration of P.A.S.-positive material. Sections stained in this way were therefore compared with sections stained by P.A.S. alone from material fixed in formaldehyde saline for 24 hours and in formaldehydesublimate-saline. This comparison showed that the reaction of material to the P.A.S. technique was much weaker in sections previously stained by the P.F.A.B. method, and cells stained by the P.A.S. technique were not easily seen.

A comparison was then made between sections treated with performic acid and stained by the P.A.S. technique and sections stained by P.A.S. alone, so that the effect of performic acid on the P.A.S. reaction might be assessed. This comparison showed that performic acid alone had the same effect as the P.F.A.B. method on the demonstration of P.A.S.-positive material. The P.A.S.-positive cells were very difficult to see in those sections treated with performic acid before staining with the P.A.S. technique (Figs. 1 and 2).
Comment.-The P.A.S. technique as a counterstain to the P.F.A.B. method appears to have little value in rats when used on material that has been suitably fixed for the demonstration of alcianblue-positive cells by the P.F.A.B. method.

Use of Buffered Performic Acid.-This problem was investigated by comparing sections stained by performic-acid-P.A.S. with sections stained by buffered performic-acid-P.A.S. and with sections stained by P.A.S. alone. The fixatives for this experiment were formaldehyde saline and formaldehyde-sublimate-saline. This comparison showed that whereas little or no P.A.S.-positive material was demonstrated in sections stained by performicacid-P.A.S., the demonstration of P.A.S.-positive material in sections stained by buffered performicacid-P.A.S. compared most favourably with that demonstrated by P.A.S. alone.

Buffered performic acid was therefore used with alcian blue and compared with the P.F.A.B. method, so that the use of buffered performic acid as an oxidizing agent could be investigated and its effect on the demonstration of alcian-bluepositive cells could be noted. This investigation showed buffered performic acid to be as effective

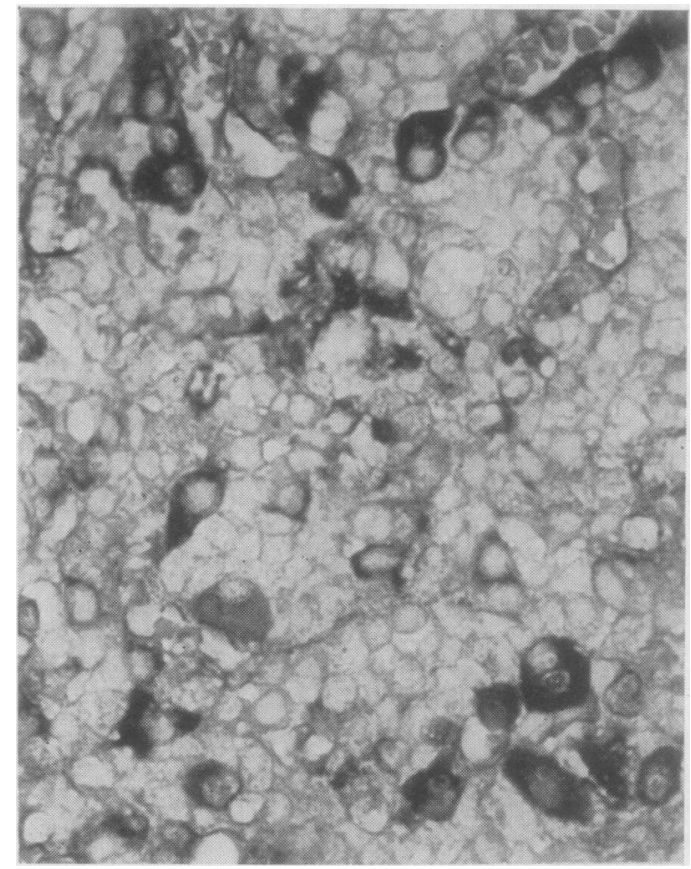

FIG. 2.-Section of same pituitary as in Fig. 1 to show strong reaction in basophils after pretreatment with buffered performic acid. P.A.S. $\times 540$. 


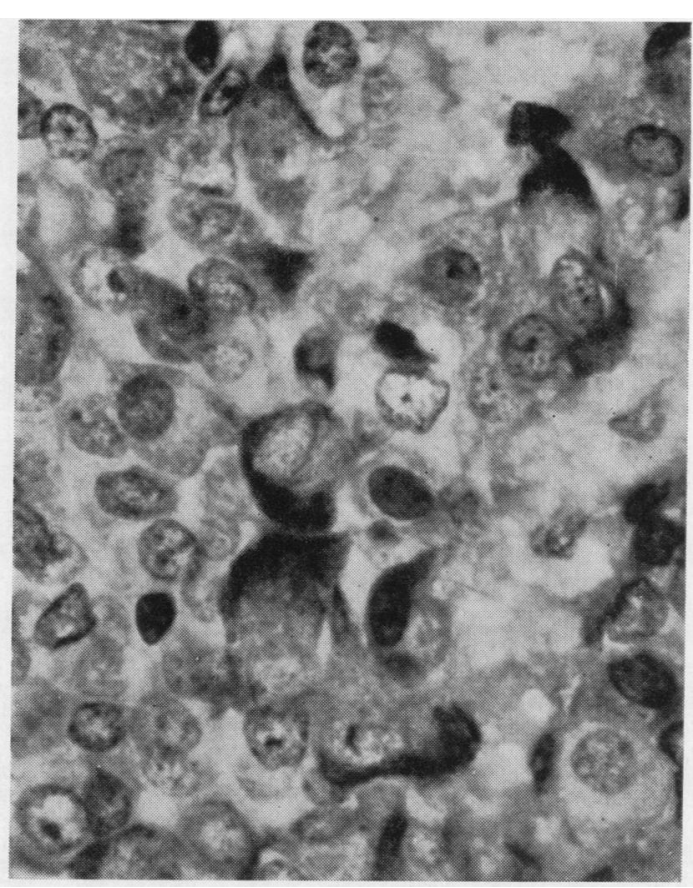

Fig. 3.-Section of rat pituitary to show thyrotrophs at centre stained witn alcian blue. Buffered P.F.A.B.-P.A.S. $\times 920$.

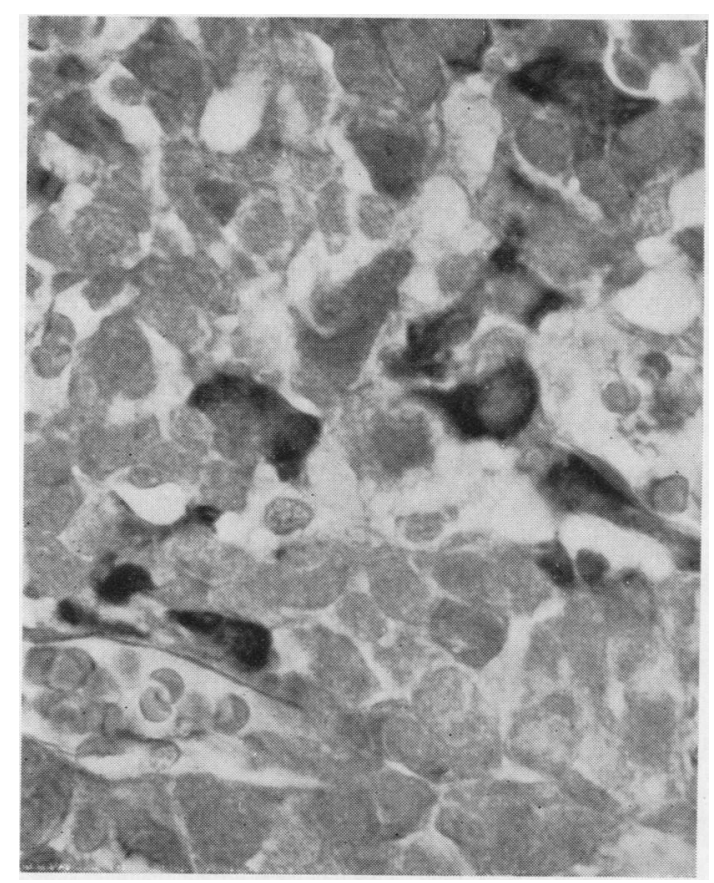

Fig. 4.-Section of same pituitary as in Fig. 3 to show parallel staining of thyrotrophs with aldehyde-fuchsin. Halmi $\times 920$. as unbuffered performic acid as an oxidizing agent in the performic-acid-alcian-blue method (Figs. 3 and 4).

Comment.-Performic acid and buffered performic acid are of equal value when used with alcian blue for the demonstration of material in certain cells.

The P.A.S. reaction is inhibited after oxidation by unbuffered performic acid but not after oxidation by buffered performic acid.

It is therefore of advantage to use buffered performic-acid-alcian-blue if the P.A.S. reaction is used as a counterstain to the P.F.A.B. method.

With regard to the human pituitary, the effect of both buffered and unbuffered performic acid on the demonstration of P.A.S.-positive mucoprotein in the (S) and (R) cells is identical.

\section{Discussion}

With regard to the preservation of the various cell components, it is of interest that P.A.S., P.F.A.B., and buffered P.F.A.B. reactions did not take place after fixation in $70 \%$ alcohol, although mucoprotein is said to be precipitated both by alcohol and by acetone (Everson Pearse, 1953b). It is clear that this material was not preserved in these experiments in a physical or chemical state necessary for the development of the P.A.S. reaction.

The present findings do, however, substantiate the view expressed by Everson Pearse (1953b) that formalin is a necessary component in any fixative designed to preserve mucoprotein and to demonstrate its presence subsequently by the P.A.S. reaction. But it should be noted that according to Elftman (1957) the combination of osmium tetroxide at $5^{\circ} \mathrm{C}$. with a subsequent chromation in potassium dichromate dissolved in mercuric chloride at $20^{\circ} \mathrm{C}$. also apparently preserves P.A.S.-positive material. This particular fixative has not been investigated in this series of experiments. However, of the fixatives which are readily available, easy to manage, and cheap, our experience suggests that, for the combined buffered P.F.A.B.-P.A.S. technique, formaldehyde saline used at room temperature for 24 hours is the most satisfactory method of fixation.

Staining with P.F.A.B.-P.A.S. Combined Method. - The P.F.A.B.-P.A.S. method, which differentiated two types of basophil cell in the human pituitary, failed to do so when applied to the rat pituitary. As it was possible to obtain positive results with the P.A.S. and the P.F.A.B. methods when applied to separate sections the effect of performic acid on the P.A.S. reaction 
was investigated and it was found that a satisfactory demonstration of P.A.S.-positive cells could not be obtained after pretreatment with performic acid, unless the performic acid were buffered. The buffered performic acid reagent was therefore incorporated with alcian blue and P.A.S. in the combined method and it was then possible to demonstrate the beta and delta types of basophil cell in rat pituitary material.

The buffered P.F.A.B.-P.A.S. method was also applied to human material and compared with the unbuffered method. It was found to give similar staining results.

The use of performic acid in effecting a differentiation of the basophil cells is paralleled by other procedures. Everson Pearse (1952) lists several techniques by means of which two varieties of basophil may be distinguished. For example, the coupled alkaline tetrazonium reaction with the application of " short-benzoylation" blockade and the Gram stain are two such methods.

The procedure which perhaps resembles the present one to the greatest degree is that described by Wilson and Ezrin (1954). In this method the P.A.S. reaction is developed and the sections first counterstained with orange $G$, then treated with phosphotungstic acid and finally stained with methyl blue. Certain P.A.S.-positive cells are purple after this procedure; the others remain red. Investigation of this method showed that the differential colour change can be produced by the action of phosphotungstic acid alone on sections stained by the P.A.S. method. This effect could easily be destroyed by washing the section in water, and reproduced by treating again with phosphotungstic acid.

The P.A.S. reaction may be affected therefore by phosphotungstic acid as well as by performic acid in such a way that two varieties of cells with different staining qualities may be differentiated. This does not imply that these effects are necessarily chemically similar, as little is known of either reaction. It also does not imply that the two cell types are necessarily functionally distinct.

The P.F.A.B. technique has frequently been used for demonstrating material relatively rich in cystine (Adams and Sloper, 1956; Adams and Swettenham, 1958; Adams and Everson Pearse, 1959) and the demonstration of centrally distributed basophil cells in the rat pituitary has been held to reflect this property. This distribution of positively reacting material, however, is in conflict with results described by Ladman and Barrnett (1956) using the dihydroxydinaphthyldisulphide (D.D.D.) method (Barrnett and Seligman, 1952, 1954). Ladman and Barrnett obtained a positive reaction in alpha cells. This discrepancy may be due to a combination of factors. Ladman and Barrnett fixed tissue in 1\% trichloracetic acid in $\mathbf{8 0} \%$ alcohol, and it is possible that the mucoprotein in beta cells may not be preserved by this procedure. Thus, as already noted above, alcohol is a particularly poor mucoprotein preservative and Meyer (1945) has claimed that trichloracetic acid is also a poor mucoprotein precipitant. It seems possible that part or all of the beta cell mucoprotein may have been lost. At the same time, D.D.D. is a very sensitive indicator of material containing cystine and a positive reaction may be given by the small quantities almost certainly present in the cytoplasm of alpha cells.

It is a pleasure to record my thanks to Professor D. S. Russell for her help, advice, and encouragement and for the facilities which she made available to me. Thanks are due also to Dr. K. Weinbren for help and criticism in the preparation of the manuscript.

\section{REFERENCES}

Adams, C. W. M., and Pearse, A. G. Everson (1959). J. Endocr., $18,147$.

and Sloper, J. C. (1956). Ibid., 13, 221.

and Swettenham, K. V. (1958).' J. Path. Bact., 75, 95.

Barrnett, R. J., and Seligman, A. M. (1952). Science, 116, 323.

(1954). J. nat. Cancer Inst., 14, 769.

Danielli, J. F. (1947). Symp. Soc. exp. Biol. (N.Y.), 1, 101.

Elftman, H. (1957). Quart. J. micr. Sci., 98, 15.

Ezrin, C., Swanson, H. E., Humphrey, J. G., Dawson, J. W., and Wilson, W. D. (1958). J. clin. Endocr., 18,917.

Fand, Sally, B. (1955). Ibid., 15, 685 .

Gomori, G. (1950). Amer. J. clin. Path., $20,665$.

Halmi, N.S. (1950). Endocrinology, 47, 289.

- (1951). Anat. Rec., 109, 300 .

(1952a). Stain Technol., 27, 61.

(1952b). Endocrinology, 50, 140.

- and Davies, J. (1953). J. Histochem. Cytochem., 1, 447.

Hotchkiss, R. D. (1948). Arch. Biochem., 16, 131.

Ladman, A. J., and Barrnett, R. J. (1956). J. Morph., 98, 305.

McManus, J. F. A. (1946). Nature (Lond.), 158, 202.

Meyer, K. (1945). Advanc. Protein Chem., 2, 249.

Pearse, A. G. Everson (1949). J. Path. Bact., 61, 195.

(1952). Ibid., 64, 791 .

(1953a). Histochemistry, p. 146. Cnurchill, London.

(1953b). Ibid., p. 24.

- (1956). J. Path. Bact., 72, 471.

Purves, H. D. and Griesbach, W. E. (1951). Endocrinology, 49, 244.

Romeis, B. (1940). In Handbuch der mikroskopischen Anatomie des Menschen, Bd. 6, Teil 3, p.99. Ed. W. von Moellendorf. Springer, Berlin.

Russell, D. S. (1956). Proc. roy. Soc. Med., 49, 1018.

Severinghaus, A. E. (1938). Ass. Res. nerv. ment. Dis. Proc., 17, 69.

Wilson, W. D., and Ezrin, C. (1954). Amer. J. Path., 30, 891 . 